\title{
reCHERches
}

Culture et histoire dans l'espace roman

4 | 2010

Les représentations du corps dans la litterature latinoaméricaine

\section{Le corps grotesque dans L'Automne du patriarche de Gabriel García Márquez}

\section{Rémi Astruc}

\section{(2) OpenEdition}

\section{Journals}

Édition électronique

URL : https://journals.openedition.org/cher/8469

DOI : $10.4000 /$ cher.8469

ISSN : 2803-5992

Éditeur

Presses universitaires de Strasbourg

Édition imprimée

Date de publication : 30 juin 2010

Pagination : 117-127

ISBN : 978-2-35410-007-0

ISSN : 1968-035X

Référence électronique

Rémi Astruc, "Le corps grotesque dans L'Automne du patriarche de Gabriel García Márquez »,

reCHERches [En ligne], 4 | 2010, mis en ligne le 15 décembre 2021, consulté le 24 janvier 2022. URL

http://journals.openedition.org/cher/8469; DOI : https://doi.org/10.4000/cher.8469

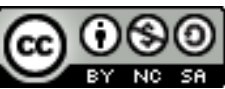

Ce(tte) œuvre est mise à disposition selon les termes de la Licence Creative Commons Attribution -

Pas d'Utilisation Commerciale - Partage dans les Mêmes Conditions 4.0 International. 


\section{Le corps grotesque dans L'Automne du patriarche de Gabriel García Márquez}

RÉmI Astruc

Université de Nancy 2

e corps occupe de toute évidence une place centrale dans L'Automne du
patriarche, ne serait-ce que parce que le roman tout entier s'organise autour de la découverte d'un cadavre, trouvé gisant dans une chambre du palais présidentiel d'un petit pays (non nommé) des Caraïbes. Il s'agit vraisemblablement du corps du dictateur, un vieillard surnommé «le général» ou encore el macho. Cette découverte sera racontée une bonne dizaine de fois au cours du roman, notamment au début de chacun des six chapitres qui découpent le récit; ce sera le point de départ de multiples évocations rétrospectives de la vie du militaire, des événements de son règne et des péripéties du pouvoir, qui seront comme autant de digressions composant en définitive le roman.

Les circonstances qui entourent cet événement sont particulièrement intrigantes : le peuple, au sortir d'une longue torpeur, a fini par pénétrer dans la résidence présidentielle après que des signes inhabituels ont suggéré une possible vacance (une vache, un beau jour, a fait son apparition au balcon du palais, puis des charognards ont brisé un matin les vitres des façades avant de s'engouffrer à l'intérieur). C'est alors qu'est découvert le cadavre en question, rendu difficilement identifiable par son état de décomposition avancée, mais qui pourrait bien être celui du père de la patrie, une figure si ancienne et depuis si longtemps en place que, devenue proprement 
mythique, sa mort apparaissait comme une chose impossible et impensable. L'incrédulité des témoins s'explique en effet avant tout par le fait que celui-ci était le détenteur d'un pouvoir qui semblait sans limites, et notamment sans limites temporelles. Le caractère extraordinaire de l'événement repose donc principalement sur cet inconcevable signe de faiblesse, voire d'échec, de la part d'un personnage jusque-là absolument omnipotent.

C'est que, le pouvoir n'existant que par des signes, le corps du chef de l'État est le signe par excellence de celui-ci, ce qui introduit le paradoxe selon lequel ce corps est tantôt l'incarnation même du pouvoir, tantôt une pure représentation, c'est-à-dire la simple trace de son absence. García Márquez explore ainsi les liens étranges qui se tissent entre le corps, le pouvoir, et l'imaginaire du corps et du pouvoir. Le roman révèle comment sur ce corps s'inscrit le mystère du pouvoir, et comment à travers lui s'exprime la dimension insaisissable et du même coup terrifiante de celui-ci. Or c'est par le recours au grotesque que l'écrivain rend visible les effets de distorsion et d'éblouissement produits par la puissance de l'imperium. Par ce corps grotesque qu'est en particulier le corps du patriarche, le lecteur pénètre en effet le système ahurissant du despotisme sud-américain. Un système tout à la fois terriblement comique et profondément inquiétant où l'on découvre en particulier que le pouvoir modèle les corps à sa convenance ${ }^{1}$, créant de ce fait un «corps-pouvoir» dont le texte déclinera les ambiguïtés. Ambiguïtés que nous analyserons en étudiant tour à tour l'hybridité du corps grotesque, les tendances contradictoires de celui-ci au redoublement et à l'effacement, enfin les rapports décisifs qui se nouent entre corps, pouvoir et imagination.

\section{Représentations grotesques du corps}

Les corps - celui, au premier chef, du dictateur, mais plus généralement de tous ceux qui gravitent autour du pouvoir - sont d'emblée présentés sur le mode de l'étrangeté et de la monstruosité. Le plus emblématique est naturellement celui du général qui est un corps incohérent et contradictoire. À l'occasion de l'une de ses très rares apparitions publiques, alors que le général parcourt les routes de son sombre royaume afin de «dispenser le sel de la santé» censé chasser la peste, voici comment il est perçu:

[...] nous vîmes apparaître dans les rues un carrosse sans fanion dans lequel personne ne surprit au premier regard le souffle glacé de la majesté du

1 Une forme donc de bio-pouvoir (Foucault) qui s'exerce avant tout sur le dictateur lui-même, plié (voire «rompu») physiquement aux exigences de l'exercice du commandement. 
pouvoir, mais à l'intérieur du velours funèbre nous vîmes les yeux létaux, les lèvres tremblantes, le gant nuptial qui jetait des poignées de sel en direction des arcades [...], nous vîmes les yeux troubles à travers les rideaux du wagon solitaire, le visage affligé, la main de jeune fille désabusée qui laissait une traînée de sel au long des hauts déserts lugubres de son enfance [...], nous vîmes les yeux de soir tombant derrière le hublot de la cabine présidentielle, nous vîmes les lèvres pâles, la main sans origine qui jetait des poignées de sel dans les villages engourdis par la chaleur... (232)

Le texte ressasse sans cesse ainsi les mêmes détails anatomiques (qui découpent le corps, celui-ci n'étant pratiquement jamais saisi dans son entier), détails saisissants que sont les «lèvres blafardes», les «mains de demoiselle", les petits yeux taciturnes ou encore - ailleurs - les pieds plats et immenses, les oreilles qui bourdonnent, le testicule hernié ayant atteint la taille d'un melon, voire l'absence de lignes de la main, traits qui composent un singulier portrait du chef de l'État. En y regardant de plus près, ce portrait singulier du dictateur obéit à une double série. Les signes de la grandeur du pouvoir (largement fantasmés) s'opposent aux marques de la réalité corporelle humaine: d'une part se trouve donc la réalité somatique d'un "vieillard crépusculaire» de taille juste moyenne, très vieux, affligé d'une hernie, souffrant de surdité et doté de pieds plats; ce sont là autant de défauts d'un corps banalement humain, en proie à la déchéance physique du grand âge. Mais d'autre part, ce portrait correspond à celui du corps fantasmé du pouvoir, reconnaissable à la taille de géant, correspondant à un être sans âge car il a tous les âges, désigné par ce corps lui-même pour gouverner et qui jouit par ailleurs - on verra comment - du don d'ubiquité tout en passant pour thaumaturge: autant de signes, dans cette seconde série, qui sont les marques incontestables de la grandeur, les caractéristiques d'un être hors du commun et proprement surhumain appelé à régner. La description qui est donnée de ce corps installe donc celui-ci dans un entre-deux qui est l'un des traits essentiels du corps grotesque. Il s'inscrit en effet à la fois entre réalité et fantasme mais aussi dans un mixte ambigu des règnes humain et animal, du masculin et du féminin, et plus encore dans un entre-deux troublant entre la vie et la mort: le dictateur apparaît pâle, décharné, grabataire mais en même temps indestructible et à jamais redoutable.

Le grotesque des corps, dans L'Automne du patriarche, s'exprime encore à travers les mauvais traitements auxquels ceux-ci sont soumis, notamment du fait de l'arbitraire d'un pouvoir sanguinaire ou à l'inverse dans des réactions tout aussi brutales et cruelles de révolte contre celui-ci. Cela engendre dans tous les cas un véritable désordre des corps, durement 
violentés dans la lutte qui entoure le pouvoir. Ce sont d'abord les corps suppliciés, dépecés, explosés des victimes du régime (l’armée se débarrassera par exemple de deux mille enfants devenus trop encombrants après avoir été utilisés pour les tricheries de la loterie nationale en les embarquant sur un bateau chargé d'explosifs) ou des rébellions contre celui-ci (des soldats mutins seront mitraillés lors d'une révolution manquée, les assassins de la famille du général seront découpés en morceaux qui seront exposés partout à travers le pays). Le corps d'un ministre félon, ancien plus fidèle compagnon gagné à la rébellion contre le patriarche insane, sera proprement consommé par les autres conjurés, apprêté en met délicieux, rôti à point et servi dans son uniforme avec une botte de persil dans la bouche - de même qu'en réaction l'épouse et le fils du général seront dévorés par des chiens affamés lâchés contre eux en plein marché. Enfin, c'est le corps maltraité et humilié du dictateur déchu, ou plutôt de sa doublure qui a été assassinée par des rebelles, offrant au général le spectacle surréaliste de la vie après sa mort et du traitement promis à sa propre dépouille:

[...] il vit le brasier allumé sur la Place d'Armes pour brûler les portraits officiels et les lithos de calendrier qu'on trouvait partout et à toute heure depuis le début de son régime, et il vit passer son propre corps qu'on tirait et qui laissait sur le pavé une traînée de décorations et d'épaulettes, de boutons de dolman, d'effilochures de brocard, une passementerie d'agrafes, de glands de sabre de jeux de cartes, et les dix soleils tristes de roi de l'univers, maman, regarde dans quel état ils m'ont mis, disait-il, en sentant sur sa propre chair l'ignominie des crachats et des pots de chambre de malades qu'on lui vidait dessus au passage du haut des balcons, horrifié à l'idée qu'il pourrait être dépecé et digéré par les chiens et les charognards au milieu des hurlements délirants et du tonnerre de la pyrotechnie pour ce carnaval de ma mort. (30-32)

C'est donc le contraste saisissant entre la sublimité du pouvoir et un corps rendu abject par ce même pouvoir qu'impose le texte. Un contraste d'autant plus marquant qu'il revient tout au long du roman à travers la comparaison obsédante du corps rutilant du général flottant sur le marasme de son empire abandonné avec un poisson ventre en l'air.

\section{Instabilité du corps grotesque}

Le corps-pouvoir est par ailleurs un corps fortement instable, glissant d'un même mouvement dans deux directions contradictoires. D'un côté celui-ci a tendance à s'effacer, à disparaître, de l'autre il manifeste une tendance à s'augmenter et à se déployer, le tout selon cette règle étrange 
qui veut qu'il disparaisse à force de proliférer, qu'il prolifère à mesure qu'il disparaît ${ }^{2}$.

Le premier mouvement correspond à l'extension de ce corps qui a tendance à déborder de lui-même: c'est ce que traduisent en particulier les différents traits du gigantisme associés à la personne du général. En effet, le corps du dictateur est d'abord monstrueux par excès, un excès qui s'inscrit de différentes manières, au premier chef par la taille démesurée qui fait passer celui-ci pour un géant aux yeux de la population, mais aussi par son âge fabuleux ou encore par cette extension extraordinaire de sa personne que constitue son immense progéniture (il aurait conçu cinq mille bâtards, tous prématurés, de ses innombrables concubines). Ce gigantisme s'illustre encore intimement et sans doute plus emblématiquement dans cette excroissance monstrueuse du testicule, qualifiée de hernie, qui est une des marques les plus sûres de l'identité du dictateur (c'est à cela que le cadavre sera identifié comme étant celui du général). En effet, cette "roupette surdimensionnée» illustre cette tendance du corps grotesque à déborder et à s'épancher sur l'extérieur, à envahir tout l'espace.

Mais l'aspect le plus troublant de cette nature envahissante du corpspouvoir réside sans doute dans la démultiplication des dépositaires du commandement (à la différence du pouvoir lui-même qui reste la propriété du général). Celui-ci est successivement abandonné entre les mains de différents lieu-tenants (Patricio Aragonès, Rodrigo de Aguilar, José Ignacio Saenz de la Barra), en vertu du principe proclamé par le général: "faites comme vous voulez, après tout qui c'est qui commande, merde alors!» (63) qui constitue l'une des expressions les plus paradoxales de l'omnipotence de celui-ci. Mais, par ailleurs, le dédoublement du dictateur se fait à un certain point effectif, celui-ci pouvant réellement apparaître dans plusieurs lieux en même temps, ce qui lui permet notamment de se faire acclamer dans les rues tandis que d'autres croient l'avoir vu en train de jouer aux dominos dans une salle du palais, ou encore de surgir bien vivant au cœur du conseil des ministres tandis que sa dépouille mortuaire est exposée dans la chapelle présidentielle. Cette ubiquité (initialement le produit d'une habile supercherie ayant consisté à transformer en doublure un sosie), qui permet

2 En cela il épouse parfaitement les deux tendances historiques du grotesque: un grotesque de la prolifération et de l'envahissement (selon un schéma rabelaisien et bakhtinien) et un grotesque de l'extinction et de la disparition (selon un modèle kafkaïen conceptualisé par Wolfgang Kayser), confirmant de fait, nous semble-t-il, la définition que nous avons proposée du grotesque comme «impossibilité réalisée». Voir notre essai (Astruc 2009). 
d'accréditer l'idée qu'il serait immortel, deviendra pourtant bientôt inutile puisqu'il sera admis de tous (pour en avoir en quelque sorte fait l'expérience) que le pouvoir, à l'instar de son enveloppe charnelle, est partout et contrôle tout.

Le second mouvement dont témoigne le corps-pouvoir est exactement inverse, puisqu'il se caractérise par la disparition progressive de la personne du général qui semble s'éteindre d'elle-même sous le poids des ans. Ainsi l'apparence du dictateur traduit cet effacement propre aux personnes de grand âge qui se terrent dans leurs appartements et sortent de moins en moins au grand air, paraissent recroquevillées par la sénilité et comme rabougries par la maladie: le général est ainsi décrit comme un «fantôme phtisique» (190 notamment) et effectivement perçu d'abord et avant tout comme une silhouette décharnée, un être vidé de sa substance dont il ne resterait plus que les contours flottants. De fait, son apparence se signale par l'extrême maigreur et l'étrange féminité qui s'exhale d'un corps travaillé par le grand âge, celle d'un être qui a pratiquement intégralement disparu pour ne plus sembler subsister que par ses mains qui pendent à la fenêtre de la limousine présidentielle où est comme avalé le reste de son corps.

L'exact contrepoint du gigantisme du personnage est donc le caractère spectral de celui-ci. Le général n'est plus qu'une ombre déambulant dans le palais, une lumière surprise à la fenêtre de l'immense bâtisse, autrement dit une simple "présence» entraperçue par les citoyens, mais une présence qui reste néanmoins parfaitement obsédante. Le corps du dictateur, incarnation du pouvoir, enveloppe charnelle de celui-ci, est pris dans un processus de désincarnation qui déréalise cet enracinement corporel jusqu'à un degré extrême. Il finit par ne plus exister ainsi que des signes fugaces et trompeurs de la réalité de ce corps et de ce pouvoir: une fenêtre éclairée dans le palais, une empreinte dans la boue, les effigies du général sur les pièces de monnaie ou les portraits accrochés dans les bâtiments officiels, et, plus ténu encore, un semblant d'ordre global qui semble attester du fait que quelqu'un doit bien être aux commandes quelque part, comme semble en témoigner la seule longévité de la fanfare municipale:

[...] aucun mortel ne l'avait aperçu depuis l'époque du vomito-negro, et pourtant nous savions qu'il était bien là car le monde continuait, la vie continuait, le courrier arrivait, la fanfare municipale jouait chaque samedi sa kyrielle de valses idiotes sous les palmiers poussiéreux et les mêmes réverbères de la place d'armes, d'autres vieux musiciens remplaçant dans l'orchestre les musiciens défunts. (8) 
La désincarnation extrême du pouvoir est là encore inversement proportionnelle à l'effet de saisissement que celui-ci est en mesure d'inspirer aux citoyens. La simple trace du pouvoir - car il ne reste en effet plus que des traces de celui-ci -, même en creux comme une empreinte de botte dans la boue, est capable de susciter émerveillement et effroi:

[...] nous restâmes pétrifiés devant le creux laissé par une grande et rude semelle qui avait l'éclat, l'autorité au repos et le relent de vieille gale d'une piste de tigre habitué à la solitude, et dans cette trace nous vîmes le pouvoir, nous sentîmes le contact de son mystère avec beaucoup plus de force révélatrice que lorsque l'un d'entre nous fut choisi pour le rencontrer en personne $[\ldots]$ (176)

L'empreinte du pied du dictateurest ainsi le signe quasi mystique de la présence absente de celui-ci - et la disparition du corps devient cause de "vertige» face au rayonnement d'un pouvoir qui subsiste et se renforce in absentia. L'absence est en effet devenue le signe même de la puissance incontestée du général.

\section{Corps, pouvoir et imagination}

C'est ainsi qu'alors qu'il infuse tout, le pouvoir paradoxalement s'abolit, ne laissant plus subsister pour l'incarner qu'un fantoche dont personne n'a plus entendu parler depuis bien longtemps. Ce dernier n'apparaît en définitive pas plus réel qu'une fable, tout juste bonne à effrayer les enfants. C'est que dans l'écheveau complexe que le pouvoir tisse avec le corps, l'imaginaire apparaît comme un rouage essentiel dont l'enjeu principal est l'inscription du pouvoir dans la durée.

Les rapports du corps et du pouvoir se tissent ainsi autour d'une étrange aporie. Le corps du dictateur est le signe et le siège du pouvoir. Or ce corps est par ailleurs le sujet de processus vitaux qui tendent naturellement à l'altérer, alors que l'idée même de pouvoir postule l'absolue invariance, et donc une forme d'éternité. C'est pourquoi, alors que le corps est soumis au vieillissement, à l'affaissement, à la décomposition, le pouvoir doit absolument s'opposer à ces formes de dégradation qui, en définitive, le nient. Pour le général, il ne saurait donc s'agir comme pour tout autre corps de devenir, mais simplement d'être, de rester, de demeurer. Aussi le pouvoir poursuit-il l'éternité, alors même que son support charnel subit la loi commune de la dégradation. S'engage ainsi une lutte proprement donquichottesque qui se solde par la crispation du pouvoir autour du seul objectif de se maintenir à tout prix, donc de n'être et de durer que pour 
lui-même - se muant par là même en pouvoir absolu et sanguinaire mais également, comme tout ce qui tente de résister au mouvement même de la vie, monstrueux et dans une certaine mesure comique ${ }^{3}$.

Le grotesque du corps-pouvoir réside en effet largement dans les efforts aussi désespérés que pathétiques qu'il met en œuvre pour s'opposer au temps, au vieillissement et à la mort, et donc en définitive pour tenter de s'imposer comme un corps immuable. Par une symétrie significative, le roman multipliera ainsi les exemples de traitements conservatoires des corps, exactement inverses à ceux (marques d'ignominie, d'infamie) qui étaient destinés à soumettre ou à détruire les opposants politiques. Il s'agit cette fois de traitements d'embellissement, de "purification" des corps sacrés du pouvoir: celui du général, de ses épouses et surtout de sa mère. Or cette immuabilité se révèle en soi monstrueuse à l'instar des techniques de conservation du corps de Bendicion Alvarado (la mère du général), détruit et rongé de son vivant par la maladie et qui sera «restauré» pour apparaître en "corps glorieux» de mère de la patrie grâce aux "pires méthodes de la taxidermie comme les animaux posthumes des musées de sciences naturelles» (147). Nier le temps par le corps est au principe même du pouvoir car celui-ci exige qu'aucune baisse de son intensité ne puisse être jamais soupçonnée. D'où la multiplication des fictions relativement grossières que le pouvoir invente pour se justifier et se maintenir ${ }^{4}$. Or ce sont là les relations qu'entretiennent naturellement le pouvoir et l'imaginaire par l'entremise du corps, comme le suggère Georges Balandier qui écrivait en effet que:

la métaphore corporelle est [...] l'instrument d'une institution imaginaire $d u$ pouvoir, et le révélateur de l'imaginaire qui fonde le pouvoir et permet de le soustraire à la loi du temps qui le dégrade, le frappe de précarité. (Balandier, 1985: 27)

L'Automne du patriarche donne ainsi à voir les ultimes conséquences de cette fiction du «corps-pouvoir» et notamment un corps monstrueux forcé

3 Ce sont en effet les analyses bien connues de Bergson qui semblent une nouvelle fois confirmées ici.

4 Il s'agit là en définitive de rien d'autre que du principe d'anthropologie politique connu en occident sous le nom de «double corps du roi», et qu'a étudié en particulier l'historien Emile Kantorowitz. Le corps glorieux du pouvoir étant nécessairement un corps immuable, celui-ci se maintient intact tant qu'il est incarné, ce qui rend nécessaire, à la mort du souverain régnant, un dédoublement de celui-ci afin d'intégrer le changement - le nouveau souverain - dans la continuité d'un pouvoir sans latence: ce que résume la formule de circonstance: «le roi est mort; vive le Roi» (Balandier, 1985). 
de se plier (voire de se distordre et même de se déchirer) aux exigences d'un pouvoir qui s'institue en niant les caractéristiques biologiques de celui-ci ${ }^{5}$. Or la conséquence de ce traitement extrême, c'est en définitive la disparition des catégories politiques de vérité et d'identité. Car tout est faux et artificiel dans cette fiction du pouvoir, ce dernier ne se développant en effet que dans la falsification des corps et la dénaturation des êtres. Le corps du pouvoir apparaît dès lors comme un corps froid, livré à la solitude et à la douleur, au calcul et à l'absence définitive de repos, comme le montre en particulier la «fabrication» du double du général, lequel n'est «formaté » pour assumer le pouvoir qu'en se vidant de toutes ses caractéristiques propres et en particulier de sa chaleureuse humanité:

[...] ce bon vivant paresseux qu'il avait été dans son négoce de marchand de miracles devint actif jusqu'à l'épuisement et marcheur infatigable, pingre aussi et rapace, il se résigna à l'amour à la sauvette et à dormir à même le sol, tout habillé sur le ventre et sans oreiller, et renonça à ses prétentions précoces d'une identité bien à lui, et à toute vocation héréditaire, à toute velléité dorée de rester petit souffleur de bouteilles. (14)

Le pouvoir se conserve donc au prix d'un dressage disciplinaire des corps qui, comme on le voit, se trouvent au besoin rééduqués pour incarner et protéger celui-ci. Pourtant, le corollaire de ce remodelage est la déréalisation de ce pouvoir et du corps même qui l'incarne, car malgré les stratégies de redoublement et d'ubiquité, c'est une impression de vide, de trompe-l'œil, une forme de néant inquiétant et risible qui se dégage du général, comme s'il s'agissait là de l'autre face - inévitable - du pouvoir absolu. Le patriarche n'apparaît en effet à ses concitoyens que comme un «tyran pour rire», une sombre farce sous laquelle ploie le pays tout entier.

Le «corps-pouvoir» se reconnaît en effet à la perte de l'identité de son support. Où qu'il apparaisse et quelles que soient les circonstances de son apparition, ce n'est jamais vraiment le général qui apparaît car il n'a en définitive plus d'identité. La falsification des corps du pouvoir les vide de leur identité et en particulier de leur identité humaine. Aussi le corps du général est-il toujours, lorsqu'il apparaît publiquement, le corps de quelqu'un d'autre puisqu'il ne coïncide plus avec l'image (inhumaine ou surhumaine) que s'en font les citoyens. C’est pourquoi lors de la veillée

5 Le transformant en un corps fantastique, détaché du réel, mais capable d'actualiser toutes les extrémités fantasmatiques d'une fiction anthropologique prise au pied de la lettre, du monstrueux au spectral, de la présence hystérique à l'absence la plus complète. 
funèbre de son sosie, son apparence n'est jamais plus éloignée de celle par laquelle il est connu:

Il était là, donc, comme s'il s'était agi vraiment de lui et pourtant ce n'était pas lui, étendu sur la table des banquets de la salle des fêtes, fastueux et efféminé comme un pape défunt parmi les fleurs [...] si franchement visible dans sa nouvelle identité posthume que pour la première fois on pouvait croire sans doute aucun à son existence réelle, pourtant personne n'était plus différent de lui que ce cadavre de vitrine qui à minuit continuait de mijoter à petit feu dans l'espace minutieux de la chambre ardente [...] (204-205)

C’est que, travaillé par le pouvoir, le général s'est départi de toute identité, laquelle fut balayée par les faux-semblants qui ont construit son image publique. Ainsi le roman se referme-t-il sur la révélation que le corps du pouvoir est un corps sans vie et, malgré tous ses efforts, promis à l'oubli comme tous les autres.

Il était condamné à ne connaître la vie que par son autre face, condamné à déchiffrer les coutures, à rectifier les fils de la trame et les points de la tapisserie d'illusion de la réalité sans soupçonner même trop tard que la seule vie supportable était celle qu'on pouvait montrer, celle que nous voyions nous de ce côté qui n'était pas le vôtre, mon général [...] (254)

Son domaine d'existence, d'où il a pourtant tragiquement pesé sur les destinées d'un pays tout entier et sur le moindre de ses habitants, est donc «l'envers de la vie», autrement dit une forme de mort qui n'est compatible qu'avec un simulacre d'existence:

[...] nous ne sûmes jamais qui il fut, ni comment il fut, ni s'il fut autre chose qu'un bobard de l'imagination, un tyran pour rire qui ne sut jamais où était l'envers et où était l'endroit de cette vie que nous aimions avec une passion insatiable et que vous n'osâtes jamais imaginer craignant d'apprendre ce que nous ne savions que trop qu'elle était difficile et éphémère mais qu'il n'y en avait pas d'autre, général, car nous savions nous, qui nous étions tandis qu'il resta lui définitivement dans l'ignorance $[\ldots](255)^{6}$

Et le roman se conclut donc sur la découverte pathétique et pourtant en un sens réconfortante que ce corps-pouvoir, si longtemps rivé aux plus hautes fonctions et aux plus grands honneurs, fut en définitive un corps malheureux, à l'inverse des corps maltraités, meurtris mais pourtant heureux des citoyens qui eurent, eux, une vie, malgré la terreur instaurée par le régime.

6 C'est nous qui soulignons le lien essentiel qui apparaît alors entre l'identité et le caractère transitoire de l'existence, de laquelle apparaît finalement exclu le corps-pouvoir qu'est la personne du général. 
$\mathrm{Au}$ final, le roman fait apparaître le règne du général comme une gigantesque hallucination collective dont émerge douloureusement le pays: «le lundi au petit jour la ville se réveilla d'une léthargie de plusieurs siècles sous une brise tiède et tendre de grand cadavre et de grandeur pourrie» dit la première phrase du texte. Le pouvoir en lui-même est donc explicitement assimilé à un corps en putréfaction et c'est l'ensemble du pays sous sa coupe qui empeste le cadavre en décomposition. Dans cette évocation particulièrement imagée de la dictature, le recours au grotesque a permis à la fiction romanesque de représenter de façon saisissante les innombrables fictions dont se nourrit le pouvoir et par lesquelles il altère les corps qui lui servent de support. Bien plus que d'être un portrait-charge, une dénonciation en règle des régimes despotiques qui ont fleuri au $\mathrm{XX}^{\mathrm{e}}$ siècle en Amérique latine, l'intérêt principal du roman, outre la poésie envoûtante de García Márquez, est de communiquer un vertige, de faire ressentir une espèce de nausée lancinante (portée par ces périodes si longues qu'elles étouffent la respiration) qui traduit l'enchantement merveilleux mais délétère de cet «autre monde» qu'est la dictature militaire.

\section{Bibliographie}

Astruc, Rémi, 2009, Le Renouveau du grotesque dans le roman du XXe siècle, essai d'anthropologie littéraire, Paris, Garnier.

Balandier, G., 1985, Le Détour, pouvoir et modernité, Paris, Fayard.

García Márquez, Gabriel, 1975, El Otoño del patriarca, Barcelone, Éditions Plaza \& Janes, traduction française de Claude Couffon pour les Éditions Grasset (1976). 